\title{
Dark Energy, Dark Matter, and the Multiverse
}

\author{
Ardeshir Irani \\ The Dark Energy Research Institute, Downey, USA \\ Email: artirani@aol.com
}

How to cite this paper: Irani, A. (2021) Dark Energy, Dark Matter, and the Multiverse. Journal of High Energy Physics, Gravitation and Cosmology, 7, 172-190. https://doi.org/10.4236/jhepgc.2021.71009

Received: October 2, 2020

Accepted: January 11, 2021

Published: January 14, 2021

Copyright $\odot 2021$ by author(s) and Scientific Research Publishing Inc. This work is licensed under the Creative Commons Attribution International License (CC BY 4.0).

http://creativecommons.org/licenses/by/4.0/

\begin{abstract}
Our third spatial dimension (3-D) is currently in the process of building the fourth spatial dimension. Dark Energy is being used to create higher dimensions of space from the first to the nth, and it is also responsible for the formation and the rotation of the fourth dimension from the point singularities of the vacuum space of the third dimension. To start the creation up to the nth dimension, $n$ ! zero dimensional singularities arise simultaneously from the $n=0$ level of the void. Once the creation of an $\mathrm{n}$ dimensional ellipsoidal matter Universe has been completed, the outward Dark Energy force ends, and then the inward force of gravity takes over to crush the $\mathrm{n}$ dimensional Universe into the center of the nth dimensional singularity of the void. The system is now in a non-equilibrium state because the entropy of the system has attained its lowest possible value. To bring the system back to equilibrium there will arise out of the nth dimensional void an antimatter Universe which will reverse the process to deposit all the Dark Energy back into the original levels of the void so that the net change in entropy of the system becomes zero. Dark Matter is the matter that exists in the fourth spatial dimension as 4-D matter spewed there through the Black Hole singularities of our third dimension. Ellipsoidal matter and antimatter Multiverses rotating in opposite directions are a natural byproduct of this new theory of Physics which also solves certain particle Physics problems of the Standard Model.
\end{abstract}

\section{Keywords}

Dark Energy, Dark Matter, Multiverse, Antimatter, Entropy, Particle Physics

\section{Background}

Dark Matter particles (WIMPS) also known as Cold Dark Matter (CDM) have not been observed experimentally in our 3-D Universe even though the existence of Dark Matter has been confirmed experimentally by Bullet Cluster and by Gravitational Lensing which will be discussed in the section on Dark Matter; and 
by the rotation curves of galaxies as shown in Figure 1.

Besides that, the Cosmological Constant Lambda $(\lambda)$ representing the vacuum energy density has no practical basis to explain Dark Energy because the vacuum energy density is 123 orders of magnitude larger than the current critical energy density for our Universe [1]. Hence $\lambda \mathrm{CDM}$ is an incorrect model of Cosmology which will also be discussed in more detail in a later section since data taken by the Planck satellite using the $\lambda \mathrm{CDM}$ model to calculate the Hubble constant $\left(H_{0}\right)$ does not give values consistent with other $H_{0}$ measuring techniques. The rotation of our 3-D universe proves that the Cosmological Principle is also an incorrect approximation used in Cosmology. A new fundamental theory of Physics has become necessary to explain Dark Matter, Dark Energy, and the experimental results of Cosmology without the use of the $\lambda \mathrm{CDM}$ model and the Cosmological Principle. This new theory brings forth the creation and annihilation of matter and antimatter Multiverses.

\section{Introducing Dark Energy}

Dark energy appears to be the dominant component of the physical Universe, yet there is no persuasive theoretical explanation for its existence or magnitude. The acceleration of the Universe is, along with Dark Matter, the observed phenomenon that most directly demonstrates that our theories of fundamental particles and gravity are either incorrect or incomplete. Most experts believe that nothing short of a revolution in our understanding of fundamental physics will be required to achieve a full understanding of the cosmic acceleration. For these reasons, the nature of Dark Energy ranks among the very most compelling of all outstanding problems in physical science. These circumstances demand an ambitious observational program to determine the Dark Energy properties as well as possible [2].

Dark Energy is a quantum effect that arises from within the void, while Dark

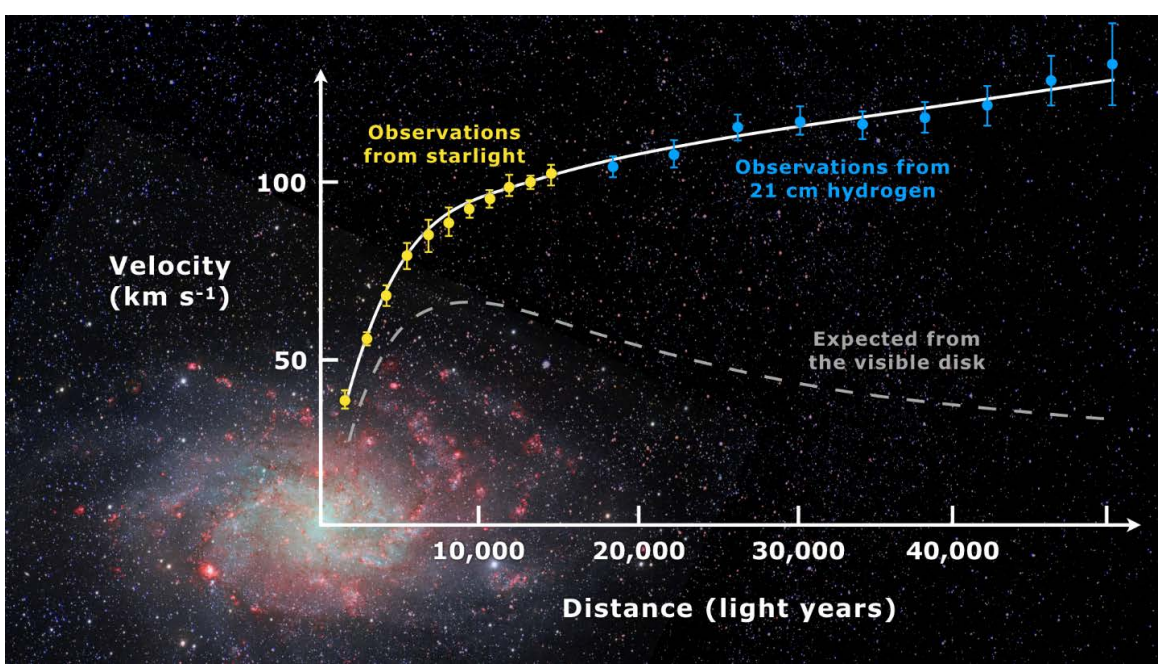

Figure 1. Velocity of stars and $21 \mathrm{~cm}$ Hydrogen gas in the rotation curve of galaxies versus what should be expected from the visible disk. 
Matter is a matter that is sent from the lower dimension to the next higher dimension that is being formed by Dark Energy. The void is a quantum region that is not the same as the vacuum of outer space. Dimensions of space can be created in the void since it contains Dark Energy in different concentrated levels of quantum numbers $\mathrm{n}$ starting with $n=0$, where the final value of $\mathrm{n}$ is determined by the total Dark Energy content of the void. The vacuum differs from the void in that the vacuum of our 3-D universe has already been created by Dark Energy since it has three dimensions of space and one dimension of time in it. The higher the quantum number $n$, the more concentrated the Dark Energy in that level, and hence the lower the entropy of that level. The lowest $n=0$ level has the highest entropy while the highest nth level that has been deposited back into the void has the lowest entropy as it will contain the sum of the Dark Energies that existed in all of the $n$ levels at the termination of the matter Multiverse in the nth level of the void.

Space and time are both intangibles, but just as time can be measured by the ticks of a clock, so too space can be measured by the distance between point masses. Space and time are not material things. They do not have a particle or wave nature associated with them. Hence space can be created faster than the speed of light without violating Einstein's theories of relativity.

Since $E=m c^{2}$ and $c=d / t$ therefore Energy $(E)$ can create matter $(m)$, space $(d)$, and time $(t)$.

Besides creating matter, Dark Energy also has the capacity to create massless particles such as real photons for which $E=p c$.

Dark Energy can also create positive and negative charges $( \pm q)$. The Energy Density in the Electric field is given by $\frac{1}{2} \in_{0} E^{2}$. From Coulomb's Law $E=q /\left(4 \pi \in_{0} r^{2}\right)$. Energy $/ \frac{4}{3} \pi r^{3}=\frac{1}{2} \in_{0}\left(q / 4 \pi \in_{0} r^{2}\right)^{2}$.

$\sqrt{\text { Energy }}= \pm q \sqrt{1 /\left(24 \pi \in_{0} r\right)}$. Hence Dark Energy can produce both positive and negative charges $+q$ and $-q$. Next, let us examine what happens when a particle and an antiparticle annihilate each other. Since Energy $=m c^{2}$ if $+q$ and $-q$ both $\rightarrow 0$ that implies $\mathrm{m} \rightarrow 0$ in accordance with the above equations, and that is exactly what happens when an electron and positron annihilate to produce two real photons of zero mass moving away with the same energy as the electron positron pair to conserve Energy.

Dark Energy can also create higher dimensions of space, one at a time, by spewing out in certain different desired directions and by combining lower dimensions of space. Dark Energy is the energy that comes out of the energy source from within the lowest $n=0$ level of the void through $n$ ! singularities, and also out of the point singularities of the vacuum space of each lower dimension creating the next higher dimension, both of which are used to create additional dimensions of space up to the nth dimension into which all matter will be deposited by a final Big Crunch.

The Cosmological Constant Lambda $(\lambda)$ is not the source of Dark Energy. 
Einstein was right to have discarded $\lambda$ calling it his life's "greatest blunder". Reinstating it in the current cosmic theory to explain Dark Energy was even a greater blunder because a $\lambda$ dominated universe would expand forever leading to the heat death of our 3-D universe.

\section{Dark Matter}

Most physicists agree that some form of New Physics (NP) must exist beyond the Standard Model-we simply do not know what it is yet. Though there are many prejudices based on preconceived ideas about the form this NP may take, it will be up to experiments at future colliders, the LHC and ILC, to reveal the true nature of this $\mathrm{N} P[3]$.

The Standard Model of particle physics cannot be extended to explain Dark Matter with Supersymmetry (SUSY) particles because Dark Matter is not a particle that exists in our 3-D universe.

Just as stars and galaxies are formed in our 3-D Universe starting with quarks, leptons, and the gauge bosons created at the very early stages of the Big Bang, and then through nucleosynthesis matter is formed from these elementary constituents taking on a 3-D shape by gravitational attraction, so too all matter takes the shape of its dimension because gravity acts in all directions of the dimension it is located in. In 4-D space gravity would act in the directions, three that we are aware of but also a fourth that we cannot picture in our 3-D minds. Matter that is sucked into the Black Holes of our third dimension stretches the space of our third spatial dimension in the direction of the fourth spatial dimension until it finally ruptures the third dimensional space as matter goes into the fourth dimension remaining close to the Black Hole and the matter of our galaxies around it. The tidal gravitational forces of the Black Hole are responsible for turning 3-D matter into its elementary constituent parts described above, which then becomes 4-D matter under the influence of fourth dimensional gravity after entering the fourth dimension. This matter in the fourth dimension that has become four dimensional is precisely the Dark Matter of our third spatial dimension.

A White Hole contains the same singularity as that of a Black Hole, however it is in the next higher dimension that is being formed by the lower dimension. While the time-reversed Black Hole which is a White Hole is a perfectly good solution of Einstein's field equations which are covariant under time reversal, we have not yet found a White Hole in our 3-D physical Universe [4]. The reason being that matter that goes into the Black Holes of our 3-D Universe enters the White Holes of the fourth spatial dimension as Dark Matter of our 3-D Universe. Since White Holes exist in the next higher dimension White Holes would have been observed in each part of the third dimension when three second dimensions were in the process of forming the third dimensions, as will be explained in the next section, but that information has been lost on the combination of the three second dimensions to complete the formation of our 3-D un- 
iverse.

Experimental proof that Dark Matter exists in the fourth spatial dimension is provided by the Bullet Cluster Experiment and the Gravitational Lensing Experiment as indicated here.

Bullet Cluster [5] as shown in Figure 2. is experimental evidence of Dark Matter in the fourth spatial dimension. The collision of two of our galactic clusters and its Dark Matter (blue colors) resulted in the separation of hot gases (red colors) from Dark Matter that passed right through itself, leaving the hot gases behind. That was because Dark Matter of our two colliding galaxies were located at different physical locations in the fourth dimension, hence they could move through each other unimpeded while the hot gases of the galaxies in our third dimension would be slowed down and left behind after the collision. To understand this effect, consider two masses colliding in the second dimension. Their corresponding Dark Matter in the third dimension located on opposite sides of the second dimension, viz. above and below the second dimensional surface, would move through each other unimpeded.

Abell 2218 is a cluster of galaxies about 2 billion light years away. Acting as a powerful lens, it distorts all galaxies lying behind the cluster core into arcs. The lensed galaxies are particularly numerous and some of them are multiply imaged. Clusters of other galaxies like Abell 2218 have also been used to infer the amount of Dark Matter.

Gravitational lensing in the galaxy cluster [6] Abell 2218 as is shown in Figure 3 should be able to confirm Dark Matter as fourth dimensional matter in the fourth spatial dimension by its gravitational effect on light in our 3-D space.

Dark Matter would distort the images of galaxies behind the cluster into arcs as it pulls some of the light being observed out of our 3-D space into 4-D space due to the curvature of space in the fourth spatial direction by the Dark Matter

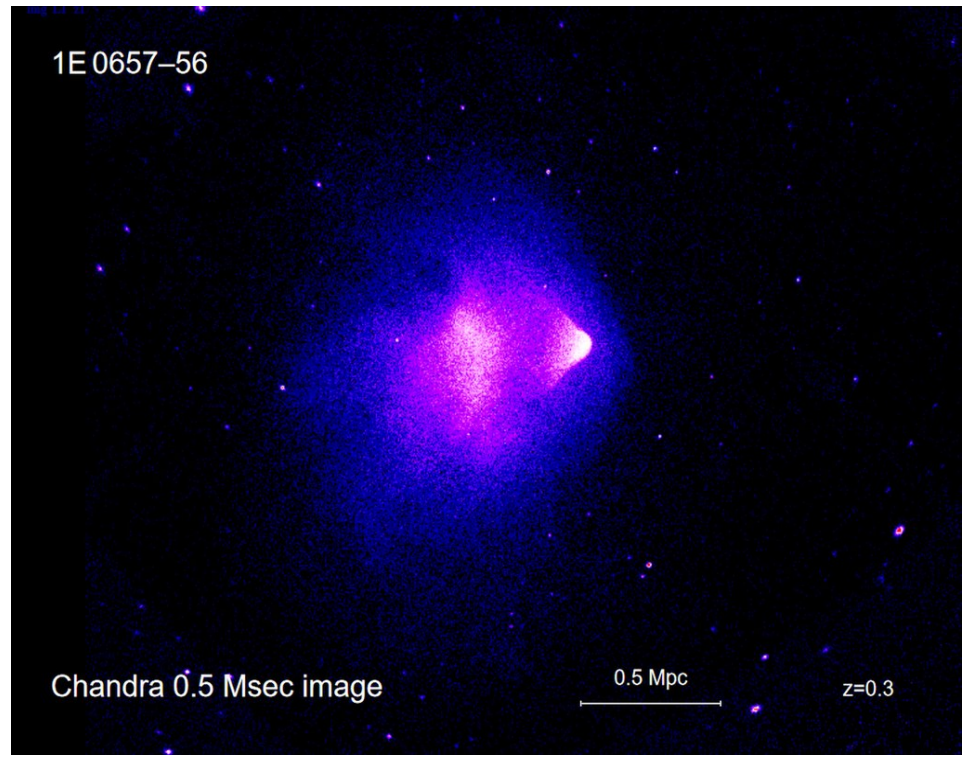

Figure 2 . The bullet cluster image taken by the chandra observatory satellite. 


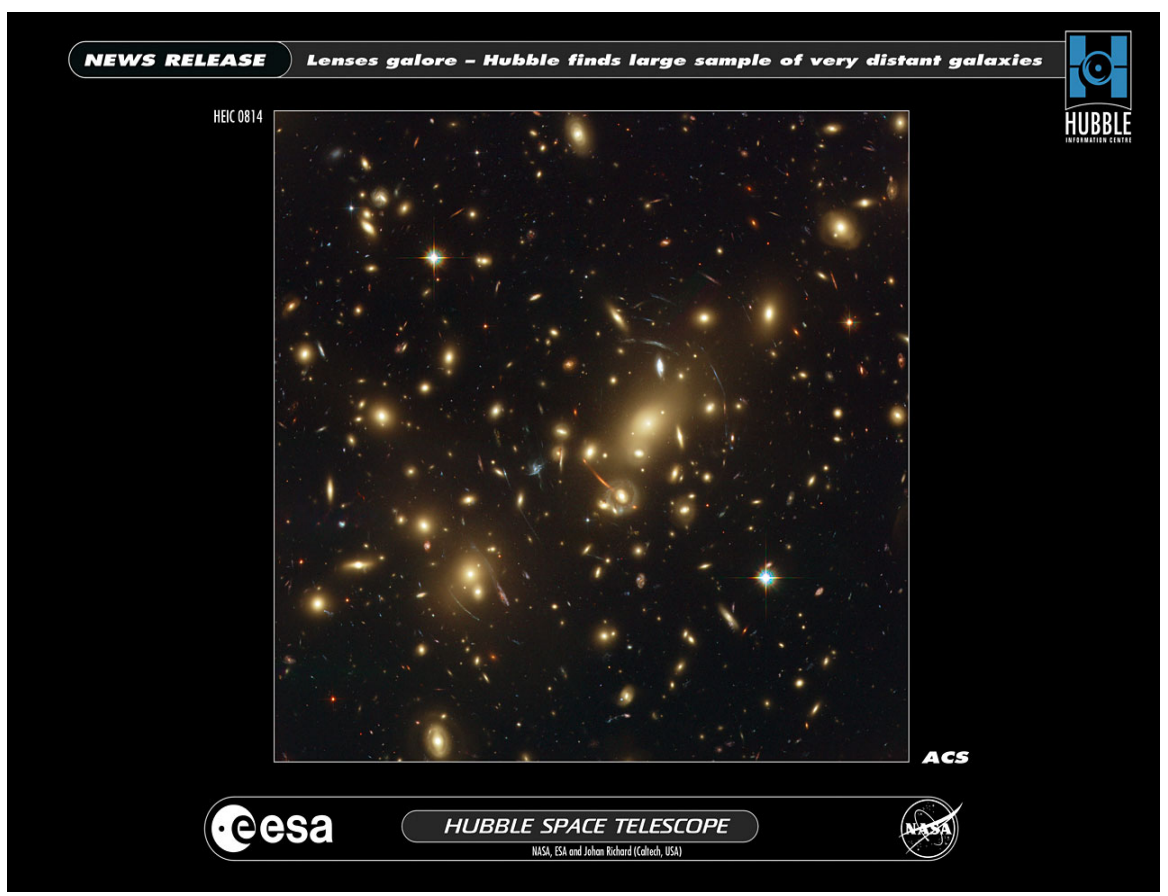

Figure 3. Gravitational Lensing observation in the galaxy cluster Abell 2218 taken by the Hubble Space Telescope.

in 4-D space. Think of a paper cut figure in the shape of a hollow circle lying on the flat surface of a 2-D table. If I lift most (but not all) of the circle figure from the surface of the table into 3-D space what will remain to be seen in 2-D space is a distorted image of the figure in the shape of an arc.

WIMPS/CDM/SUSY do not exist in our 3-D Universe to interact with ordinary 3-D matter by the weak or gravitational force. Instead it is 4-D matter that is interacting gravitationally with 3-D matter, and that is the reason for unsuccessful detection results of Dark Matter particles in our 3-D Universe by all the different experiments that have been carried out to date.

\section{Iteration and the Creation of Higher Dimensions}

For a n-dimensional (n-D) sphere of radius $R$, its volume $V$ and surface area $S$ are given by:

$$
\begin{gathered}
V(n)=\pi^{(n / 2)} R^{n} / \Gamma(1+n / 2) \\
S(n)=n \pi^{(n / 2)} R^{n-1} / \Gamma(1+n / 2) \\
V(n) / S(n)=R / n
\end{gathered}
$$

where $\Gamma$ represents the Gamma Function.

For a n-dimensional (n-D) cube of length $L$,

$$
\begin{gathered}
\mathrm{V}(\mathrm{n})=L^{n} \\
S(n)=2 n L^{n-1} \\
V(n) / S(n)=L / 2 n .
\end{gathered}
$$


Comparing the spherical and cubic shapes:

$V(1) / S(1)=R / 1$ and $L / 2-$ A circle has one start and the same end point while a straight line has two different end points.

$V(2) / S(2)=R / 2$ and $L / 4$ - Two circles come together to form a hollow 2-D sphere while four straight lines join together to form a square.

$V(3) / S(3)=R / 3$ and $L / 6$ - Three hollow 2-D spheres come together to form a 3-D sphere while six squares are joined together to form a cube.

$V(4) / S(4)=R / 4$ and $L / 8-$ Four $3-\mathrm{D}$ spheres come together to form a $4-\mathrm{D}$ sphere while eight cubes are joined together to form a 4-D cube.

$V(n) / S(n)=R / n$ and $L / 2 n-n,(n-1)$-D spheres come together to form a n-D sphere while $2 n,(n-1)$-D cubes are joined together to form a $n$ - $D$ cube.

How do we know that our Universe has a spherical and not a cubic shape? It is because the spherical solution of Einstein's field equations by Karl Schwarzschild correctly predicts the existence of Black Holes, gravitational lensing, and the precession of Mercury's perihelion. This is also experimental proof that our 3-D Universe is not flat, and that it has a spherical shape.

$n$ ! point singularities are created simultaneously in the void from level $n=0$ at the beginning of the creation process. Let's analyze the manner in which one of the $n$ ! point singularities begins to build higher dimensions with the understanding that the same process occurs simultaneously for all of the $n$ ! point singularities.

To create the first dimension in the form of a circle around the second dimensional void the point singularity shoots Dark Energy from level $n=0$ in two separate directions. The energy shot out in the first direction creates matter while its reaction force accelerates the point singularity in the opposite direction creating vacuum space between the matter and the point singularity. The energy shot out in the second direction is pointed away from the center of the circle to be formed. Having a reaction force in the direction of the center of the circle it does the work to change the direction of the point singularity from linear to circular motion since it does not have enough energy to create matter. A ring shaped 1-D space made of matter and vacuum is created within the void.

To create the second dimension around the third dimensional void two circles would start at the location of the North and South poles, each circle forming a hollow hemisphere coming together to complete the formation of a hollow sphere. Each circle shoots Dark Energy from level $n=1$ of the void from all point singularities within its vacuum in two separate directions, the first toward the center of the circle and the second in the opposite direction to the center of the hollow sphere being formed. The energy shot out in the first direction forms matter within the circle, while its reaction force accelerates the circle in the opposite outward direction creating vacuum space between the matter and circle. The reaction force of the energy shot out in the second direction does the work to change the direction of the circle upwards from the South Pole and downwards from the North Pole creating two hollow hemispheres. As the two circles approach the equatorial plane, they have opposite linear accelerations that crash 
together to cancel each other's motion as the two hollow hemispheres are joined to form a hollow sphere around the third dimensional void. A hollow 2-D spherical space made of matter and vacuum is created within the void.

Since the two circles creating the second dimensional (2-D) hollow sphere started at two locations of the third dimension, three 2-D hollow spheres creating the third dimension will start at three locations of the fourth dimension. The three hollow spheres will accelerate toward one another as all three hollow spheres come together to complete the formation of the third dimension around the fourth dimensional void. A 3-D spherical space made of matter and vacuum is created within the void. We cannot imagine the shape of the 3-D spherical space because its starting location is at three different positions of the fourth spatial dimension and it engulfs the void of the fourth dimension within its volume. The crashing cancellation of the opposite accelerations of the three hollow 2-D spheres coming together is what constitutes the Big Bang creation of our 3-D Universe.

While four third dimensions (of which our 3-D Universe is one) are creating the fourth dimension (4-D), the four third dimensions will accelerate toward one another. Only by being a part of 3-D space can we know the shapes of 1-D and 2-D spaces. Hence only after becoming a part of 4-D space which has not yet been completed is it possible to ascertain the shape of our 3-D Universe.

By the iteration process the nth dimension is formed by $(n-1)$ dimensions at $\mathrm{n}$ locations of the $(n+1)$ dimension that will come together to complete the nth dimension around the $(n+1)$ dimensional void. The accelerations of the $(n-1)$ dimensions having opposite directions will cancel one another at the completion of the nth dimension. Dark Matter of the $(n-1)$ dimensions is the matter that goes into the White Holes of the nth dimension through the Black Hole singularities of the $(n-1)$ dimensions.

Just as the lower dimensions have become part of our 3-D space and will expand as our 3-D Universe expands, so too all lower dimensions that have become part of the $(n-1)$ dimensions will expand along with the expansion of the $(n-1)$ dimensions due to the Dark Energy outward force from the vacuum space of the $(n-1)$ dimensions. All matter (living and non-living) located in the nth dimension will have taken the shape of the nth dimension just as matter in our 3-D Universe has taken a third dimensional shape. There is no evidence for a preferred center of our 3-D spatial Universe [7] which might have been the site of a Big Bang explosion. The reason for that is all spatial dimensions, starting with the first and ending with the nth, are formed around the void of the next higher dimension. All spatial dimensions are being formed without a center.

\section{Some Misconceptions in the Current Cosmic Theory}

From the dipole distortion of the CMB (Cosmic Microwave Background) temperature [8], which is a Picture of the birth of our 3-D Universe as shown in Figure 4. 


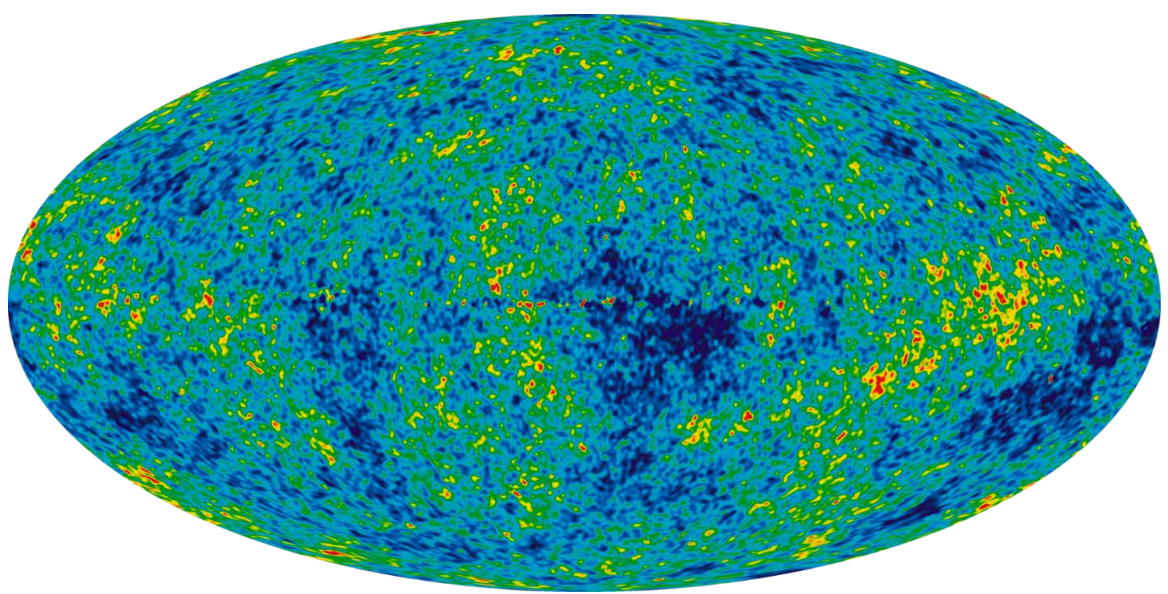

Figure 4. The black body form and the anisotropy of the Cosmic Microwave Background radiation.

The blackbody spectrum is shifted to higher temperatures on the right and to lower temperatures on the left. This implies that our 3-D baby Universe was given a rotational spin in the westerly direction; counterclockwise as viewed from above. This dipole distortion of the CMB due to the Doppler shift has been explained away incorrectly as peculiar velocity to preserve the isotropic and homogeneous nature of the CMB for the Cosmological Principle and the Robertson-Walker Matrix to be valid. According to the peculiar velocity theory, the Planck satellite that took the most detailed of the CMB pictures while moving with the earth also moved around the sun which moved around the Milky Way galactic center which moved around the center of the Local Group of galaxies moving in the general direction of the constellation Hydra. This reflects that the very massive Virgo Cluster at the center of our Local Supercluster is being gravitationally attracted to and accelerating toward the Hydra-Centaurus Supercluster. After subtracting the dipole distortion due to peculiar velocity, the remaining temperature fluctuations were used to prove that our 3-D Universe was flat using the altered CMB data which is also an incorrectly calculated result since it uses the $\lambda \mathrm{CDM}$ model, the use of which has also been made to calculate the value of the Hubble Constan $t$ [9] $\left(H_{0}\right)$. Data obtained from nearby galaxy measurements $\left(H_{0}=73 \mathrm{Km} / \mathrm{s} / \mathrm{Mpc}\right)$ by several different methods such as SHOES, Carnegie and HOLiCOW, and data from the CMB Planck picture $\left(H_{0}=\right.$ $67 \mathrm{Km} / \mathrm{s} / \mathrm{Mpc}$ ) do not agree. The experimental error bars between these two separate methods do not overlap. This discrepancy throws a monkey wrench in $\lambda \mathrm{CDM}$ as the standard model of Cosmology as also in the Cosmological Principle.

The first dimension of our Universe had a circular Euclidean shape, and the second dimension had a non-Euclidean hollow spherical shape with positive curvature (it was not flat). The current value of the density parameter determined to be $\Omega_{0} \cong 1$ for a flat universe is incorrect. This implies that the percentage of Dark Energy (68\%) that has been calculated, based on $\Omega_{0} \cong 1$ cannot be correct. 
The Schwarzschild metric, a spherical solution of Einstein's field equations indicates that our $3 \mathrm{D}$ Universe has a spherical shape, as is also the case in the Newtonian theory of gravity, and in this new theory of Physics, hence $\Omega_{0}>1$.

The Inflation theory of the early Universe which has not been confirmed experimentally is incorrect because it was designed to solve the Horizon problem and the Flatness problem. The Horizon problem is solved because the CMB black body radiation is not the same in all directions [8]. The Flatness problem is also solved because our $3 D$ Universe is not flat $\left(\Omega_{0}>1\right)$.

With the creation of each higher dimension a new phase begins to start up since all matter takes the shape of that higher dimension. The estimated age of 13.8 Billion years by the current cosmic theory can only be the present age of our 3-D Universe after its creation was completed by three of the two dimensional hollow spheres crashing together. It was named the Big Bang by Fred Hoyle, one of the proponents of the competing steady state theory of Cosmology. 13.8 Billion years does not consider the time for creating the first and the second dimensions and it is not the age of the Multiverse which will be discussed in detail in the following two sections. We can calculate the total age of our 3-D Universe since the ratio of Dark Matter to Baryonic Matter is estimated to be about 5 or 5.25 to 1 . This implies that our 3-D Universe has advanced toward about $84 \%$ completion of the fourth dimension. At the current age of 13.8 Billion years, in another 2.6 Billion years there will be a phase change to end the 3-D and begin the 4-D Universes. This implies that our 3-D Universe will have had a total lifetime of 16.4 Billion years. Quasars formed earlier in time were more active in our 3-D Universe as indicated by data from radio telescopes because there was more 3-D Baryonic matter during the early development stage of our 3-D Universe to produce active Black Holes. Our 3-D Universe will be bereft of Baryonic Matter just before it becomes part of the 4-D Universe. Each of the lower dimensions will send Dark Matter through their Black Holes into the White Holes of the next higher dimension. When all the lower dimensions come together to form the highest dimension their matter will have become part of that highest nth dimension.

As soon as Dark Energy ceases, so too will end the creation of higher dimensions. Gravity's inward pull for a closed n-dimensional Universe will begin to reverse the creation process with all the matter, now part of the nth dimension, deposited back into level $\mathrm{n}$ of the void as Dark Energy in its most concentrated form. However, the question of the stability of such a system remains because gravity is an inward force which will have to be counteracted by the outward centrifugal force once the creation of each dimension has been completed. A rotation will have to be imparted on the system as will be discussed in the section on the Rotating Ellipsoidal Multiverse. Without a rotational spin the process would have ended with the first dimension collapsing back into the void.

Since our 3-D Universe could not be created without a rotation denying the anisotropy of the $\mathrm{CMB}$ radiation by using the peculiar velocity theory was a bla- 
tantly misleading falsehood.

\section{Matter Multiverse}

The fifth space-time dimension was first included in the Kaluza-Klein Theory and then extended to multiple extra dimensions in String Theory [10].

The total number of dimensions that can be formed by the Universe is limited by the total Dark Energy content of its void. In order to prove that to create a n-dimensional sphere, a start would have to be made with $n$ ! zero dimensions, note that for the creation up to the first dimension, 1 or 1 ! zero dimensional points are required. For the creation up to the second dimension, 2 first dimensions or $2 \times 1=2$ ! zero dimensional points are required. For the creation up to the third dimension, 3 second dimensions or $3 \times 2 \times 1=6$ or 3 ! zero dimensional points are required, and by iteration for the creation up to the nth dimension, $n$ ! zero dimensional points will have arisen simultaneously in predetermined locations within the void so that the final result will be a single n-dimensional sphere surrounding the $(n+1)$ dimensional void. For $n=10$, taken from M-theory spatial dimensions which is the most current of the String theories, the Dark Energy from $10 !=3,628,800$ singularities would be needed, although as stated earlier the total number of dimensions that can be created would be determined by the total Dark Energy content of the void. The additional dimensions are not curled up or compacted as in String theory [11], instead they have yet to be created by the Dark Energy within the void of these higher dimensions.

Dark Energy concentration increases in quantum mechanical steps within the void. Dark Energy that comes out of the vacuum space of the $(n-1)$ dimensions to create the nth dimension will have more concentrated energy by a factor of $n$ ! than the Dark Energy that comes out of the $n=0$ level of the void because matter in the nth dimension is more concentrated by $n !$. Since the first dimension is created by Dark Energy from quantum number level $n=0$, the nth dimension will be created by the Dark Energy from quantum number level $(n-1)$ of the void. The Dark Energy of each level in the void is in thermal equilibrium with less concentrated energy or higher entropy in the $n=0$ level than in level $\mathrm{n}$, and the Dark Energy of each level is thermally isolated from all the other levels. By mixing the Dark Energy of all the levels, not only has the entropy of the system been lowered but also the system is no longer in thermal equilibrium. Both these effects are responsible for the non-equilibrium of the final level $\mathrm{n}$ of the void into which all the Dark Energy is deposited.

When one zero-eth dimension has created the first dimension there will remain $n ! / 1 !=n$ ! first dimensions. When two first dimensions have created the second dimension there will remain $n ! / 2 !=n ! / 2$ second dimensions. When three second dimensions have created the third dimension there will remain $n ! / 3 !=$ $n ! / 6$ third dimensions. Hence $n ! / 6$ third dimensions would be currently in the process of creating the fourth dimension. When $n,(n-1)$ dimensions have created the nth dimension there will remain $n ! / n !=$ one nth dimension. For $n=$ 
10 , our 3-D Universe would currently be one out of $10 ! / 3 !=3,628,800 / 6=$ 604,800 third dimensional Universes. We do not know the current value of $n$ with absolute certainty because we do not know the total energy content within the void, but what we do know is that our 3-D is not an isolated Universe. Multiverse is a natural byproduct of the new theory of Physics.

\section{A Rotating Ellipsoidal Multiverse}

Since rotational motion changes the shape of a planet from spherical to ellipsoidal, I extend the $\mathrm{n}$-dimensional spherical shape to a n-dimensional ellipsoidal shape. Both shapes have positive curvature. The iteration formula for $V(n) / S(n)$ remains the same [12], since it is proportional to $1 / n$ for both $n$-dimensional spheres and $\mathrm{n}$-dimensional ellipsoids.

Since everything including galaxies, black holes, stars, planets, and sub-atomic particles rotate, this implies that our 3-D Universe is also rotating as indicated earlier from CMB data that shows that our baby 3-D Universe was born with a rotational spin. The advantage of giving each dimension a spin is to keep it from collapsing on itself due to the inward gravitational force. The outward centrifugal force is made to be greater than the inward gravitational force by the rotational motion thereby flinging galaxies in the outward direction. This rotational motion is responsible for the experimentally [13] observed outward acceleration of our 3-D Universe.

To examine how this rotational motion is generated consider the point singularities of the vacuum space of the first dimensional circle that is being formed around the second dimensional void shooting Dark Energy in the direction of the circle. The point singularities are pushed in the opposite direction of the additional matter and vacuum space that is being formed, but the additional matter created pushes the matter that already exists in the forward direction. There now exists a one dimensional moving space made of matter and vacuum that is circulating, the space and hence the ellipse that the circle is being transformed into by the addition of more matter and vacuum space is therefore rotating, with the axis of rotation going through the center of the ellipse in the direction of the third dimensional void. This process of creating a 1-D rotating ellipse by adding Dark Energy from the $n=0$ level of the void does not take place separately but occurs simultaneously while the formation of the 1-D circle is taking place.

Two ellipses rotating in the same direction start at the North and the South poles. Since they are rotating in the same direction, they will give the hollow ellipsoid being formed a rotation with the axis of rotation starting at the North and the South poles of the third dimension and joining at the center of the third dimensional void. As Dark Energy is added from the point singularities of level $n=1$ through the vacuum spaces of the second dimension in the direction of rotation, the 2-D hollow ellipsoid that is being formed will continue to rotate about an axis going through its North and South poles.

Three hollow 2-D ellipsoids rotating in the same direction will start at three 
locations of the fourth dimension to form a 3-D rotating ellipsoid with their axes of rotation going from each of their three starting locations in the fourth dimension to the center of the fourth dimensional void. As Dark Energy is added from the point singularities of level $n=2$ through the vacuum spaces of the third dimension in the direction of rotation, the 3-D ellipsoid that is being formed will continue to rotate about its axis of rotation.

Four rotating 3-D ellipsoids (of which our 3-D Universe is one) will start at four locations of the fifth dimension to form a 4-D rotating ellipsoid with their axes of rotation going from their four starting locations to the center of the fifth dimensional void. As Dark Energy is added from the point singularities of level $\mathrm{n}=3$ through the vacuum spaces of the fourth dimension in the direction of rotation, the 4-D ellipsoid that is being formed will continue to rotate about its axis of rotation.

By iteration, $\mathrm{n}$ rotating $(n-1)$ dimensional ellipsoids will start at $\mathrm{n}$ locations of the $(n+1)$ dimension to form a $n$-dimensional rotating ellipsoid with their axes of rotation going from their $\mathrm{n}$ starting locations to the center of the $(n+1)$ dimensional void. As Dark Energy is added from the point singularities of level $(n-1)$ through the vacuum spaces of the nth dimension in the direction of rotation, the n-D ellipsoid that is being formed will continue to rotate about its axis of rotation.

Just as Dark Energy from level $n=0$ starts the creation and the rotation of the first dimension, so too Dark Energy from the final level $\mathrm{n}$ of the void stops the rotation leading to the gravitational collapse of the nth dimension into the final level $n$ of the void. The process of rotation is halted by adding Dark Energy from level $n$ through the vacuum spaces of the nth dimension in the opposite direction of rotation.

The ellipsoidal shape implies that the Multiverse is closed and hence has a finite size.

A rotating Universe cannot be isotropic because it has a preferred direction which is the direction of the axis of rotation. The further out you go from the axis of rotation the faster you will be rotating as in a merry-go-round. Rotation also tends to fling galaxies in the outward direction away from the axis of rotation due to the centrifugal force. Hence our Universe is neither isotropic nor homogeneous. Since the Cosmological Principle is not valid, we cannot use the same space-time equations for different parts of the Universe. The other problem within the current cosmic theory arises because the scale factor $a(t)$ used therein was made to further simplify the space-time equations by allowing distances to expand as a function only of time. Introducing a $(t)$ means using comoving coordinates which means working within the framework of the expansion of space only without regard to gravitational and rotational effects.

\section{Antimatter Multiverse}

CRT is a symmetry where $\mathrm{C}$ (charge conjugation) stands for matter or antimat- 
ter charge, R stands for rotation clockwise or counterclockwise, and $\mathrm{T}$ stands for time moving forward or backward. According to the Feynman diagram the electron-positron annihilation process leads to the creation of two photons as shown in Figure 5.

Matter $\left(e^{-}\right)$time running in the forward direction is the same as antimatter $\left(e^{+}\right)$time running in the backward direction. Hence time running forward in the matter Multiverse would be the same as time running backward in the antimatter Multiverse. Time reversal is like watching a movie backwards from end to start. In such a movie if an object is rotating clockwise then as you rewind the movie that same object would be rotating counterclockwise. CRT and CPT symmetries are similar but not identical since the mirror image of clockwise rotation is counterclockwise rotation, but we do not need the aspect of Parity that includes the inversion of left to right and up to down.

CRT like CPT symmetry preserves the laws of Physics for both the matter and the antimatter Multiverses.

The matter Multiverse takes Dark Energy from its higher entropy states ( $\mathrm{n}=0$ to $n-1)$ and deposits the Dark Energy back into the nth dimension of the void in the lowest entropy state. Because of the non-equilibrium state of the system the antimatter multiverse brings out the Dark Energy of the nth dimension void as nth dimensional space with $\mathrm{n}$-dimensional antimatter in it and then begins to rewind itself depositing the Dark Energy in each lower level in the same concentrated form as it was removed by the matter Multiverse. The antimatter in each level is sent from the White Holes of that higher level into the Black Holes of the next lower level before the higher dimension is demolished, thereby antimatter taking the dimensional form of the lower level.

Starting with one $\mathrm{n}$-dimensional antimatter Universe there will remain $\mathrm{n},(n-$ 1) dimensions; and then $\mathrm{n} \times(n-1),(n-2)$ dimensions; and then $n \times(n-1) \times$ $(n-2),(n-3)$ dimensions; and by the iteration process, $n \times(n-1) \times(n-$ 2) $\ldots \ldots . .3 \times 2 \times 1=n$ ! zero dimensions. When $n=1$, all the remaining Dark Energy in the 1-D antimatter space is sent back into the $n=0$ void by the $n$ ! separate point singularities. We considered earlier how in a matter multiverse two ellipses rotating in the same direction would form a hollow rotating ellipsoid. A hollow antimatter ellipsoid rotating in the opposite direction as the matter ellipsoid would turn the hollow antimatter ellipsoid into two antimatter ellipses. Since the matter ellipses were formed with a counterclockwise spin as viewed

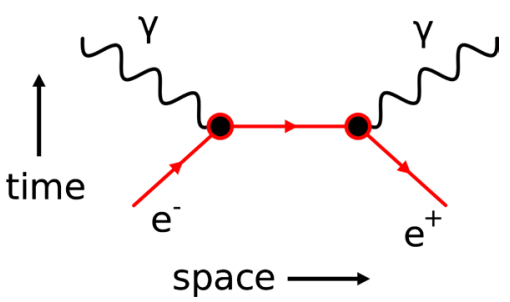

Figure 5. The Feynman diagram for electron-positron annihilation creating two real photons. 
from above, in conjunction with our 3-D Universe having a counterclockwise spin as shown earlier, a clockwise spin would send the antimatter ellipses back into the void through the $n$ ! point singularities.

\section{Entropy}

The entropy within the void exists in quantum mechanical steps of decreasing entropy from levels $n=0$ to a final $n$. The matter Multiverse takes all the Dark Energy from each of these levels and deposits it into the $n$th dimensional level $n$ of the void in the lowest entropy state. The entropy of our 3-D Universe is not increasing as is the current belief, but it is decreasing because Dark Matter in 4-D space is 3-D matter that is being removed from our 3-D Universe. The entropy of each higher level being formed is lower because matter in each level is being concentrated by $n$ ! thereby reducing the amount of disorder (entropy) of the Multiverse. The lowest entropy state that has been deposited into the nth level of the void is in a non-equilibrium state, and this is what drives the antimatter Multiverse to deposit all the Dark Energy back into their original levels of the void.

Upon completion since the process is cyclic, the net change in entropy of the system is zero, and the system is stable once more because the system is now in its highest possible entropy state.

The difference between matter and antimatter is that matter starts at the highest entropy level to begin the non-equilibrium state of the system by decreasing its entropy while antimatter starts at the lowest entropy level to begin to restore the equilibrium of the system. In fact, an asymmetry between matter and antimatter has already been observed in accelerator experiments such as BaBar at SLAC in California and Belle at KEK in Japan where the decay rates of the short lived $B^{0}$ mesons are different for matter and antimatter mesons. This solves the Baryogenesis problem since the asymmetry between matter and antimatter explains why our 3-D universe is made of matter only. The three conditions for Baryogenesis as first stated by the Russian physicist Andrei Sakharow in 1967 are satisfied by the new theory. The three conditions are: Baryon non-conservation, particle-antiparticle asymmetry in the laws of physics, and non-equilibrium. The currently accepted Baryogenesis theory of a slight imbalance between matter and antimatter just after the Big Bang is incorrect because there is no known theory or experiment for the origin of the quark-antiquark or the electron-positron asymmetry in the early part of our 3-D Universe due to the annihilation of all the antiquarks and positrons. Although antiquarks and positrons can be created in accelerators and by cosmic rays, yet there is no evidence of them being annihilated in large numbers during the early stages of our 3-D matter Universe.

Experiments conducted at electron-positron colliders such as the B Factory at the SuperKEKB collider (Belle II detector) in Tsukuba, Japan, taking entropy into consideration should provide further proof of the asymmetry between matter and antimatter due to entropy effects as indicated by the new theory of Phys- 
ics. Researchers at SuperKEKB will be able to compare their results with those from $\mathrm{LHCb}$ at CERN, which has been tracking B decays amid the shrapnel of proton-proton collisions. With such different approaches to B-meson production, the two experiments complement each other nicely. LHCb researchers have the advantage of higher energy and more $\mathrm{B}$ decays to analyze; the cost is having to sift through scores of unrelated particles that are not created in the relatively clean electron-positron collisions at SuperKEKB. One method of decreasing the entropy of the electron-positron or the proton-proton beams would be to compress them in the lengthwise/radial directions (increasing their luminosity) just before the two beams collide with each other and then observing how the asymmetry of the products differ from the uncompressed beams after collisions. Since the entropy of the compressed beams would be lower the decay rates of their products should show a larger ratio of antiparticles to particles compared to the uncompressed beams. The reason it is more difficult to form higher luminosity beams is because they have lower entropy, therefore they tend to become more unstable.

\section{Certain Particle Physics Problems of the Standard Model have been Solved}

A) Why are there only three families of fundamental particles and why is only the first family stable?

A.) There are only three families because we live in a 3-D spatial Universe and each dimension adds an extra family to the list. Only the first family is stable because it is made from Dark Energy of the $n=0$ level which has the highest entropy. As Dark Energy from the higher levels get mixed together the entropy of the system is lowered producing a non-equilibrium state, and hence it is responsible for the particles of each higher level becoming more and more unstable.

B.) Why is the neutrino only left-handed, meaning that it advances with a clockwise spin?

B.) Since our 3-D universe is rotating in the counterclockwise direction the neutrinos inertia gives it a clockwise spin. This confirms that the neutrino has mass. This also means that the antineutrino is right-handed since the antimatter universe spins in the opposite direction as the matter universe. The neutron must also be left-handed and the antineutron right-handed.

C.) A new Conservation Law along the same lines as the Conservation Laws of Baryon Number, Lepton Number and Strangeness Number emerges, called the Conservation of Spin.

C.) The Conservation of Spin states that during a natural decay process the spin number on the left side of the equation must equal the spin number on the right side of the equation. Giving clockwise or left-handed spin the number +1 and counterclockwise or right-handed spin the number -1, consider the spontaneous decay of the neutron. $n(l) \rightarrow p(l)+e^{-}(l)+\bar{v}_{e}(r)$. The left side of the equation has spin number +1 while the right side of the equation has spin num- 
ber $+1+1-1=+1$. This implies that during this decay process the spins of the neutron, the proton and the electron must both be clockwise or left-handed. Next consider the decay of a muon. $\mu^{-} \rightarrow e^{-}+\bar{v}_{e}+v_{u}$. Since the neutrino and the antineutrino have opposite spins whose spin numbers cancel, this implies that both the muon and the electron must have the same spin, either left-handed or right-handed. These theoretical predictions of the new Conservation Law can be tested experimentally in existing particle accelerators.

\section{Conclusions}

Without rotation our Universe could not have been created. Hence denying the experimental existence of anisotropic $\mathrm{CMB}$ radiation and also the outward acceleration of our 3-D universe due to rotation, and stating that our 3-D Universe is flat even though we have known since the time of Newton that it had a spherical shape is a great injustice being done to the advancement of the theory of Cosmology, and brings forth the need of the new Physics.

The idea of a Multiverse-an ensemble of Universes-has received increasing attention in Cosmology as the outcome of the originating process that generated our own Universe [14]. If there is one thing history has taught us, it is that we should expect the unexpected. Just as the sixteenth-century Polish astronomer Copernicus proposed correctly that our earth revolved around the sun and hence it was not at the center of the Universe; so too this NP proves that our 3-D Universe is not at the center of the Multiverse, but rather it is only one among hundreds of thousands of other 3-D Universes. We hold no special privilege within the Multiverse creation and annihilation process.

The cited Dark Energy problem should, in principle, also be achieved in the framework of extended gravity [15] or the quantum theory of gravity since Dark Energy is a quantum effect.

Just as scaffolding is a structure that is taken down upon the completion of the building it was used to build, so too dimensions of space, time, and matter/antimatter are structures that are dispensed off with once all the Dark Energy has been deposited back into the original quantum mechanical levels of the void where space, time, and matter/antimatter do not exist.

\section{Conflicts of Interest}

The author declares no conflicts of interest regarding the publication of this paper.

\section{References}

[1] Ryden, B. (2017) Introduction to Cosmology. 2nd Edition, Cambridge University Press, Cambridge, 66, 67.

[2] Albrecht, A., Bernstein, G., Cahn, R., Freedman, W.L., Hewitt, J., Hu, W., Huth, J., Kamionkowski, M., Kolb, E.W., Knox, L., Mather, J.C., Staggs, S. and Suntzeff, N.B. (2006) Report of the Dark Energy Task Force.

[3] Rizzo, T.G. (2004) Pedagogical Introduction to Extra Dimensions. SLAC Summer Institute, Menlo Park. https://doi.org/10.2172/833088 
[4] Ta-Pei-Cheng (2015) A College Course on Relativity and Cosmology. Oxford University Press, Oxford, 141.

https://doi.org/10.1093/acprof:oso/9780199693405.001.0001

[5] Ta-Pei-Cheng (2015) A College Course on Relativity and Cosmology. Oxford University Press, Oxford, Figure 8.4, 166.

https://doi.org/10.1093/acprof:oso/9780199693405.001.0001

[6] Binney, J. (2016) Astrophysics: A Very Short Introduction. Oxford University Press, Oxford, Figure 32, 108.

https://doi.org/10.1093/actrade/9780198752851.001.0001

[7] Pebbles, P.J.E. (1993) Principles of Physical Cosmology. Princeton University Press, Princeton, 6.

[8] Ryden, B. (2017) Introduction to Cosmology. Second Edition, Cambridge University Press, Cambridge, Figure 8.2, 144-146.

[9] Astronomy Magazine (2019) Tension at the Heart of Cosmology. 20-27.

[10] Shiffman, M. (2009) Large Extra Dimensions: Becoming Acquainted with an Alternative Paradigm. World Scientific, Singapore. https://doi.org/10.1142/9789814304030_0001

[11] Randall, L. and Sundrum, R. (1999) Large Mass Hierarchy from a Small Extra Dimension. Physical Review Letters, 83, 3370-3373.

https://doi.org/10.1103/PhysRevLett.83.3370

[12] John Wilson, A. (2009) Volume of n-Dimensional Ellipsoid. Sciencia Acta Xaveriana, 1, 101-106.

[13] Ta-Pei-Cheng (2015) A College Course on Relativity and Cosmology. Oxford University Press, Oxford, 224, 225.

[14] Ellis, G.F.R. and Stoeger, W.R. (2004) Multiverses and Physical Cosmology. Monthly Notices of the Royal Astronomical Society, 347, 921-936. https://doi.org/10.1111/j.1365-2966.2004.07261.x

[15] Corda, C. (2009) Interferometric Detection of Gravitational Waves: The Definitive Test for General Relativity. International Journal of Modern Physics D, 18, 2275-2282. https://doi.org/10.1142/S0218271809015904 


\section{Appendix: Article Text}

\section{Introduction}

Twelve Key Features of the new theory of Physics:

1) Experimental proof exists that Dark Matter is in the fourth spatial dimension as 4-D matter.

2) Experimental proof exists that $\lambda \mathrm{CDM}$ is not the standard model of Cosmology, and that the Cosmological Principle is an incorrect approximation.

3) Experimental proof exists that $\Omega_{0}>1$ and hence our third dimensional universe is not flat, and the $68 \%$ of Dark Energy calculated based on $\Omega_{0}=1$ is incorrect.

4) Experimental proof exists that our 3-D Universe is currently accelerating outwards and that it is rotating as indicated by the new theory.

5) Experimental proof exists of an asymmetry between matter and antimatter as indicated by the new theory.

6) The Flatness problem, the Horizon problem, and the Baryogenesis problem have been solved by the new theory.

7) The entropy of our 3-D Universe is not increasing as is the current belief, but it is decreasing because Dark Matter in 4-D space is 3-D matter that is being removed from our 3-D Universe.

8) The current Inflation theory, peculiar velocity theory, and the Baryogenesis theory are incorrect. The new theory of Physics corrects them.

9) An experiment based on entropy considerations has been proposed at accelerator facilities such as LHCb at CERN and the SuperKEKB collider in Japan for further proof of the new theory.

10) The theory of ellipsoidal matter and antimatter Multiverses rotating in opposite directions emerges from known physical principles.

11) Certain particle Physics problems of the Standard Model have been solved by the new theory of Physics.

12) Dark Energy can create matter $(m)$, space $(d)$, time $(t)$, real photons, and positive and negative charges $( \pm q)$. 